\title{
ON THE PROBLEM OF TIME-HARMONIC WATER WAVES IN THE PRESENCE OF A FREELY FLOATING STRUCTURE
}

\author{
N. KUZNETSOV
}

To V. M. Babich on the occasion of his 80th birthday

\begin{abstract}
The two-dimensional problem of time-harmonic water waves in the presence of a freely floating structure (it consists of a finite number of infinitely long surface-piercing cylinders connected above the water surface) is considered. The coupled spectral boundary value problem modeling the small-amplitude motion of this mechanical system involves the spectral parameter, the frequency of oscillations, which appears in the boundary conditions as well as in the equations governing the structure's motion. It is proved that any value of the frequency turns out to be an eigenvalue of the problem for a particular structure obtained with the help of the so-called inverse procedure.
\end{abstract}

\section{§1. INTRODUCTION}

This paper deals with coupled boundary value problems describing the irrotational motion of an inviscid, incompressible, heavy fluid (water) in which a structure consisting of a finite number of rigid bodies is floating freely. Water extends to infinity in the horizontal directions as well as downwards and is bounded above by a free surface, thus modeling an infinitely deep open sea. The surface tension is neglected on the free surface and the coupled motion of the structure and water is assumed to be of small amplitude near equilibrium, which allows us to use a linear model. We restrict ourselves to considering structures formed by infinitely long horizontal surface-piercing cylinders that are rigidly connected with each other above the water surface (an infinitely long pontoon is a typical example). This assumption leads to another simplification that consists in studying only the case of two-dimensional motion that is the same in every plane orthogonal to the structure's generators.

The time-dependent equations for the two-dimensional mechanical system described above are formulated in Subsection 2.1. A full discussion of the three-dimensional coupled boundary value problem was given by John in his pioneering work 1 (see also the paper 2] by Beale, who presented the equations of motion in a more convenient matrix form). In Subsection 2.2, we turn to the case where the water waves are time-harmonic, as well as the motion of the structure, and the so-called external forces (they, for example, are due to constraints on the structure motion) are absent. We also discuss conditions that must be imposed on the behavior of a time-harmonic solution at infinity. The resulting problem is the coupled spectral problem involving the spectral parameter, the frequency of oscillations, which appears in the boundary conditions as well as in the equations of

2010 Mathematics Subject Classification. Primary 76B15, 76B03, Secondary 35Q35, 35P05.

Key words and phrases. Coupled spectral problem, time-harmonic water waves, freely floating structure, trapped mode.

The author is indebted to Dr. O. Motygin for stimulating discussions and to Professor S. Nazarov for his comments on the first version of the paper. 
the structure's motion. We restrict ourselves to considering only the explicit form of this spectral problem, which is convenient for our purpose of constructing an example of a structure floating freely and trapping time-harmonic water waves. However, it is possible to reformulate the problem as an operator spectral problem in an appropriate Hilbert space (see [2] for further details).

It may appear surprising that no rigorous results were obtained for the problem formulated in Subsection 2.2 after 1950, when John published his second classical work [3] dealing with water waves. (The paper contains, in particular, the uniqueness theorem in which rather restrictive assumptions are made about both the structure's geometry and the frequency of motion.) The reason for this is the difficulty of the problem, which motivated attempts to use a model in which only heave motion of a freely floating structure is allowed; see, for example, the papers [4] and [5]. Their topic was the construction of trapped-mode solutions in the framework of this simpler model. However, in those papers, the question was addressed by means of heuristic arguments only.

On the other hand, the paper [3] laid the foundation for studies of the problem that describes time-harmonic water waves in the presence of a fixed structure. The progress achieved in this direction was presented in detail in the book [6. Part 1]. In particular, much attention was given to the existence of trapped modes in the case of a fixed surfacepiercing structure (the groundbreaking paper [7] on this topic was published by M. McIver in 1996).

In the present paper, we use a special trapped-mode solution for a fixed structure (see [8] and [6, \$4.2.2.3]) in order to construct a particular solution to the coupled timeharmonic problem in the case of a freely floating structure. Up to the present, nothing was known about the solvability of the latter problem (the question remained untouched in [3]), and our aim in the present paper is to fill in this gap at least partially. We prove that any value of the frequency turns out to be an eigenvalue of the problem for a particular structure obtained with the help of the so-called inverse procedure.

Exaggerating slightly, this procedure can be described as follows. Instead of finding a solution of a problem in a given domain, we deal with a domain reasonable from a hydrodynamic point of view that must be found for a given solution. It is interesting to observe that it was L. Euler who proposed to apply this method in hydrodynamics 250 years ago. The problem considered in his paper [9] is similar to that in the present paper insofar as the two problems involve the same equation and boundary conditions, but there is also a fundamental distinction. Euler's problem - it is referred to as the sloshing problem nowadays (see the survey paper [10] for a historical review beginning with the cited work of Euler) — is posed in a bounded domain occupied by water (a container) and serves for determining the eigenfrequencies and eigenmodes of free oscillations of water in it. On the other hand, a water domain is unbounded in the problem considered here.

\section{§2. Statement of PRoblems}

2.1. The time-dependent problem. The coupled equations of motion are formulated under the following geometric assumptions. Water at rest occupies a domain whose cross section orthogonal to the structure's generators is

$$
W=\mathbb{R}_{-}^{2} \backslash S, \text { where } \mathbb{R}_{-}^{2}=\{(x, y): x \in \mathbb{R}, y<0\},
$$

and $S$ denotes the submerged part of the structure's cross section in the equilibrium position. All of the bodies forming the immersed part of the structure are surfacepiercing, so that the interior of $S$ is the union of a finite number of bounded, simply connected domains in $\mathbb{R}_{-}^{2}$. They are adjacent to the $x$-axis, and their closures have no 
common points. Let the cross section of the entire structure (including its part above water) be the closure $\widehat{S}$ of a single domain, and so $S=\widehat{S} \cap \mathbb{R}_{-}^{2}$.

By $B=\partial S \cap \mathbb{R}_{-}^{2}$ we denote the wetted boundary of $S$, and $F=\partial W \backslash \bar{B}$ is the cross section of the free surface at rest. We suppose that the closure $\bar{B}$ consists of curves formed by a finite number of $C^{2}$-arcs, so that $W$ has no cusp points on $\partial \mathbb{R}_{-}^{2}$. The latter requirement is imposed because otherwise a contribution to the spectrum might emerge that is not related to water waves (see Nazarov and Taskinen [11 for the details).

Now we turn to the problem itself; it is based on a linear model because the motion is assumed to be small-amplitude. We recall that, in accordance with the standard linearization procedure (see [1, §2] and [6, Introduction]), all unknowns are proportional to a small nondimensional parameter $\epsilon$ (in fact, expansions of unknowns in powers of $\epsilon$ are used). In the linearized problem, the boundary conditions are imposed on $\partial W$.

The assumptions that the water motion is irrotational and two-dimensional imply that there exists a velocity potential in $\bar{W}$, provided the domain $W$ is simply connected, which is the case under our geometric conditions. If $\epsilon \Phi(x, y ; t)$ is the first-order velocity potential, which means that the velocity field is equal to $\epsilon \nabla \Phi(x, y ; t)$ at every instant $t$ $\left(\nabla=\left(\partial_{x}, \partial_{y}\right)\right.$ is the spatial gradient $)$, then the continuity equation implies that

$$
\nabla^{2} \Phi=0 \text { in } W \text { for all } t .
$$

The standard linear boundary condition on the free surface involves the dependence of the velocity potential on the time variable $t$ and has the following form:

$$
\Phi_{t t}+g \Phi_{y}=0 \text { on } F \text { for all } t .
$$

Here $g>0$ is the acceleration due to gravity that acts in the direction opposite to the $y$-axis. Relation (2) is a consequence of Bernoulli's equation and the kinematic condition. Both of them are taken linearized; the first expresses the fact that the pressure is constant on $F$, and the second means that there is no transfer of matter across $F$.

Let $\left(q_{1}^{(0)}, q_{2}^{(0)}\right)$ be the $(x, y)$-coordinates of the center of mass of the structure at rest. In order to specify the near-equilibrium position of the structure, we use a vector $q=\left(q_{1}, q_{2}, q_{3}\right)$ whose components are bounded functions of $t$. Indeed, we describe by $\epsilon\left(q_{1}, q_{2}\right)$ the first-order displacement of the center of mass from its rest position in the $(x, y)$-plane, while $\epsilon q_{3}$ gives the first-order angle of rotation about the axis that goes through the center of mass orthogonally to the $(x, y)$-plane (the angle is measured from the $x$-axis to the $y$-axis).

It is clear that the time derivative $\dot{q}$ characterizes the motion of the structure in the following way: $\epsilon\left(\dot{q}_{1}, \dot{q}_{2}\right)$ is the velocity vector of the translational motion and $\epsilon \dot{q}_{3}$ is the angular velocity. Then the linearized kinematic condition on the wetted boundary of the structure is as follows:

$$
\frac{\partial \Phi}{\partial n}=\dot{q} \cdot N=\sum_{1}^{3} \dot{q}_{j} N_{j} \quad \text { for } \quad(x, y) \in B \quad \text { and all } t,
$$

where

$$
\left(N_{1}, N_{2}\right)=\left(n_{x}, n_{y}\right) \text { and } N_{3}=\left(x-q_{1}^{(0)}, y-q_{2}^{(0)}\right) \times\left(n_{x}, n_{y}\right)
$$

are the unit normal directed out of $W$ and the moment of the normal about the center of mass, respectively. In order to specify the behavior of $\Phi$ near corner points of $\partial W$ and taking conditions (2) and (3) into account, we require that $\Phi$ belong to the Sobolev space $H_{\mathrm{loc}}^{1}(W)$.

The three equations of motion of the structure can be written in the form

$$
E \ddot{q}=-\int_{B} \Phi_{t} N \mathrm{~d} s-g K q+\frac{f}{\rho} \text { for all } t .
$$


Here $\rho$ is the density of water, and $f=\left(f_{1}, f_{2}, f_{3}\right)$ has the following components: $\epsilon\left(f_{1}, f_{2}\right)$ is the first-order net external force applied to the structure per unit span; $\epsilon f_{3}$ is the firstorder moment of the force about $\left(q_{1}^{(0)}, q_{2}^{(0)}\right)$. Finally, the $(3 \times 3)$-matrices $E$ and $K$ are defined as follows:

$$
E=\left(\begin{array}{ccc}
I^{M} & 0 & 0 \\
0 & I^{M} & 0 \\
0 & 0 & I_{2}^{M}
\end{array}\right) \text { and } K=\left(\begin{array}{ccc}
0 & 0 & 0 \\
0 & I^{D} & I_{x}^{D} \\
0 & I_{x}^{D} & I_{x x}^{D}+I_{y}^{S}
\end{array}\right)
$$

and their entries are

$$
\begin{aligned}
I^{M} & =\rho^{-1} \int_{\widehat{S}} \mathrm{~d} m>0, \quad I_{2}^{M}=\rho^{-1} \int_{\widehat{S}}\left[\left(x-q_{1}^{(0)}\right)^{2}+\left(y-q_{2}^{(0)}\right)^{2}\right] \mathrm{d} m>0, \\
I^{D} & =\int_{D} \mathrm{~d} x>0, \quad I_{x}^{D}=\int_{D}\left(x-q_{1}^{(0)}\right) \mathrm{d} x, \\
I_{x x}^{D} & =\int_{D}\left(x-q_{1}^{(0)}\right)^{2} \mathrm{~d} x>0, \quad I_{y}^{S}=\int_{S}\left(y-q_{2}^{(0)}\right) \mathrm{d} x \mathrm{~d} y .
\end{aligned}
$$

Here $\mathrm{d} m$ is the element of the mass distribution within the structure per unit span; $D=\partial \mathbb{R}_{-}^{2} \backslash \bar{F}$ is the part of the $x$-axis within the structure. Thus, the entries of $E$ and $K$ depend on the total mass of the structure and on its various moments. It is obvious that $E$ is positive definite and $K$ is symmetric. Since only the $(2 \times 2)$-submatrix $K^{\prime}$ that stands in the lower right corner of $K$ has nonzero elements, the first equation of system (44) is independent of the other two equations.

The statement of the problem must be augmented by the following:

- Archimedes' law expressed by $I^{M}=\int_{S} \mathrm{~d} x \mathrm{~d} y$;

- the equality $I_{x}^{S}=0$, where $I_{x}^{S}=\int_{S}\left(x-q_{1}^{(0)}\right) \mathrm{d} x \mathrm{~d} y$;

- the condition that $K^{\prime}$ is positive definite.

The second of these requirements means that the centers of buoyancy and mass are on the same vertical; this condition together with the first one guarantees the equilibrium of the rest position. The third condition implies that the equilibrium position of the structure is stable, which follows from the results formulated in [1, §2.4]. (Stability is understood in the usual sense: an instantaneous, infinitesimal disturbance causes the position changes that remain infinitesimal, except for purely horizontal, for all subsequent times.) Indeed, an assertion in [1] has the following consequence for the two-dimensional case.

Proposition 1. There is an orthogonal change of coordinates such that the condition that $K^{\prime}$ is positive definite takes the following form. If the structure's center of mass lies above that of displaced water, then the distance between the two centers does not exceed the ratio

$$
\frac{\text { moment of inertia of } D \text { about the origin }}{\text { area of } S}
$$

This inequality is the classical stability condition presented, for example, in [12, Chapter XXXI].

Finally, we note that relations (1)-(4) must be complemented by proper initial conditions in order to obtain a well-posed initial-value problem (see 2, §3], where this question was discussed in detail). However, our aim is to study the steady-state motion simple harmonic in time, and so not depending on the initial conditions (the way in which the corresponding hydrodynamic phenomenon is conceived was described in [3, p. 46]). Therefore, we do not go into the details of the initial conditions. 
Some simple properties of system (44). We suppose that the structure we deal with has the following two properties. It floats freely, that is, $f=0$ for all $t$ in system (4), and it is symmetric. The latter means that:

(i) the structure's cross section is symmetric about the $y$-axis (both the submerged part $S$ and the part above water);

(ii) the structure's mass distribution is the same in every cross section and is symmetric about the $y$-axis.

Condition (ii) immediately implies that $q_{1}^{(0)}=0$, and condition (i) shows that $I_{x}^{D}=0$ and also allows us to investigate separately the velocity potentials that are even and odd functions of $x$, that is, either

$$
\Phi(x, y ; t)=\Phi(-x, y ; t)
$$

or

$$
\Phi(x, y ; t)=-\Phi(-x, y ; t)
$$

for all $t$. In the next assertion, we show that a substantial simplification of system (4) follows for symmetric structures from condition (6).

Proposition 2. Let a freely floating structure $S$ be symmetric. If a solution of the timedependent water-wave problem for $S$ has the first component satisfying condition (6), then system (4) defining this solution takes the form

$$
\begin{aligned}
& I^{M} \ddot{q}_{1}=0, \quad \ddot{q}_{3}+\omega_{3}^{2} q_{3}=0, \\
& I^{M} \ddot{q}_{2}+g I^{D} q_{2}=-\int_{B} \Phi_{t} n_{y} \mathrm{~d} s .
\end{aligned}
$$

In the second equation in (8), the coefficient $\omega_{3}^{2}=\omega_{3}^{2}\left(q_{2}^{(0)}\right)$ is positive and equals

$$
g \rho\left[\int_{D} x^{2} \mathrm{~d} x+\int_{S}\left(y-q_{2}^{(0)}\right) \mathrm{d} x \mathrm{~d} y\right] / \int_{\widehat{S}}\left[x^{2}+\left(y-q_{2}^{(0)}\right)^{2}\right] \mathrm{d} m .
$$

Proof. Since the relations $q_{1}^{(0)}=0$ and $I_{x}^{D}=0$ follow from the assumption that $S$ is symmetric, we have

$$
K^{\prime}=\left(\begin{array}{cc}
I^{D} & 0 \\
0 & I_{x x}^{D}+I_{y}^{S}
\end{array}\right)
$$

which immediately yields equation (19) because the structure $\widehat{S}$ is floating freely $\left(f_{2}=\right.$ 0 ). Moreover, the requirement that $K^{\prime}$ be positive definite (it was imposed in order to guarantee the stability of equilibrium) implies that

$$
I_{x x}^{D}+I_{y}^{S}=\int_{D} x^{2} \mathrm{~d} x+\int_{S}\left(y-q_{2}^{(0)}\right) \mathrm{d} x \mathrm{~d} y>0
$$

and so the quantity $\omega_{3}^{2}=g\left(I_{x x}^{D}+I_{y}^{S}\right) / I_{2}^{M}$, given by formula (10), is positive.

Furthermore, conditions (i) and (6) yield the formulas

$$
\int_{B} \Phi_{t} n_{x} \mathrm{~d} s=0 \text { and } \int_{B} \Phi_{t} N_{3} \mathrm{~d} s=\int_{B} \Phi_{t}\left[x n_{y}-\left(y-q_{2}\right) n_{x}\right] \mathrm{d} s=0,
$$

the second of which follows from the fact that $q_{1}^{(0)}=0$. Indeed, both of the integrands are odd functions of $x$ and the integrals do vanish because $B$ is symmetric about the $y$-axis. Hence, the first and third equations of system (4) reduce to equations (8) for a structure floating freely $\left(f_{1}=f_{3}=0\right)$. 
Remark 1. From the hydrodynamic point of view, Proposition 2 means that the force due to the hydrodynamic pressure has no horizontal component and produces no rolling moment on a symmetric structure provided the velocity potential satisfies condition (6).

An immediate consequence of equations (8) is the following statement.

Corollary 1. Let a freely floating structure be symmetric. If a solution of the timedependent water-wave problem has the first component satisfying condition (6), then $q_{1}$ vanishes identically and

$$
q_{3}=a_{3} \sin \left(\omega_{3} t+\alpha_{3}\right)
$$

where $a_{3}, \alpha_{3} \in \mathbb{R}$ and $\omega_{3}$ is given by formula (10).

Proof. A solution of the first equation (8) is a linear function of $t$, the coefficients of which must vanish because otherwise the conditions that $q$ is bounded and the structure is symmetric are violated. Since the coefficient of $q_{3}$ in the second equation (8) is positive, formula (11) gives the general solution of this equation.

Remark 2. By Corollary 1, a symmetric two-dimensional structure may execute the periodic roll motion with the radian frequency $\omega_{3}$. However, this is impossible for structures consisting of a single body and satisfying the so-called John condition (no point of $B$ lies below $F$ ), provided $\omega_{3}$ has the following properties. It is sufficiently large and coincides with the frequency of water waves that are also assumed to be time-harmonic. Indeed, under the conditions listed the coupled problem has only a trivial solution: this follows from the uniqueness theorem of John [3] (see Remark 3 below).

2.2. The coupled time-harmonic problem. Now we formulate the problem of timeharmonic water waves coupled with a similar motion of a freely floating structure. We represent the periodic oscillations of the structure's position in a form similar to (11):

$$
q(t)=\zeta \sin (\omega t+\alpha), \quad \text { where } \zeta \in \mathbb{R}^{3}, \alpha \in \mathbb{R}, \text { and } \omega>0 .
$$

Taking into account the boundary condition (3) and the equations of motion (4), we see that the Ansatz for the velocity potential with the same radian frequency $\omega$ must be as follows:

$$
\Phi(x, y ; t)=\phi(x, y) \cos (\omega t+\alpha), \quad \text { where } \phi \in H_{\mathrm{loc}}^{1}(W) .
$$

Substituting representations (12) and (13) into relations (11)-(4) with $f=0$, we obtain the coupled problem for the pair $(\phi, \zeta)$ :

$$
\begin{aligned}
\nabla^{2} \phi & =0 \quad \text { in } \quad W ; \\
\phi_{y}-\frac{\omega^{2}}{g} \phi & =0 \quad \text { on } \quad F ; \\
\frac{\partial \phi}{\partial n} & =\omega \zeta \cdot N \text { on } B ; \\
\omega^{2} E \zeta-g K \zeta & =-\omega \int_{B} \phi N \mathrm{~d} s .
\end{aligned}
$$

Viewing the parameter $\omega$ as unknown, we get a spectral problem, which must be completed with a condition describing the behavior of $\phi$ at infinity.

The most general way to introduce such a condition is to complexify problem (14)(17), that is, to assume that $\zeta \in \mathbb{C}$ and $\phi$ is complex-valued. Then $\Phi$ and $q$ can be obtained as follows (cf. [3, p. 45]):

$$
\Phi(x, y ; t)=\operatorname{Re}\left\{\mathrm{e}^{-i(\omega t+\alpha)} \phi(x, y)\right\}, \quad q(t)=\operatorname{Re}\left\{i \zeta \mathrm{e}^{-i(\omega t+\alpha)}\right\} .
$$


After complexification, it is natural to augment problem (14)-(17) by the radiation condition (see [3, Introduction] and [6, §3.2.1.2]):

$$
\lim _{\sigma \rightarrow+\infty} \sum_{ \pm} \int_{-\infty}^{0}\left|\phi_{|x|}( \pm \sigma, y)-i \frac{\omega^{2}}{g} \phi( \pm \sigma, y)\right|^{2} \mathrm{~d} y=0
$$

here $\sum_{ \pm}$denotes the sum of two terms corresponding to $x= \pm \sigma$. This condition means that waves behave at large distances like progressive waves outgoing from the structure.

Remark 3. It is worth demonstrating how simple the proof of the uniqueness theorem of John is (see Remark 2) when the kinematic condition on $B$ and the equations of motion are written in the form (16) and (17), respectively. Indeed, if a structure consists of a single surface-piercing cylinder subject to the geometric condition of John, then Lemma II in 3 shows that relations (14), (15), and (18) yield

$$
\int_{B} \frac{\partial \phi}{\partial n} \bar{\phi} \mathrm{d} s>0 \text { unless } \phi \equiv 0 \text { in } \bar{W} \text {. }
$$

Using condition (16) in the relation obtained as the inner product of $\zeta$ and the relation complex conjugate to (17), we obtain

$$
\omega^{2} \zeta \cdot E \bar{\zeta}-g \zeta \cdot K \bar{\zeta}=-\int_{B} \frac{\partial \phi}{\partial n} \bar{\phi} \mathrm{d} s
$$

Since the matrix $E$ is positive definite (see the first formula (5) for its diagonal form), the last identity contradicts inequality (19) if $\omega$ is sufficiently large, unless the pair $(\phi, \zeta)$ is trivial.

We turn to constructing an example of a structure floating freely in time-harmonic water waves. For this, it suffices to use a condition at infinity that is simpler than (18); namely, we shall require that

$$
\phi(x, y)=O\left(\left[x^{2}+y^{2}\right]^{-1 / 2}\right), \quad|\nabla \phi(x, y)|=O\left(\left[x^{2}+y^{2}\right]^{-1}\right) \quad \text { as } \quad x^{2}+y^{2} \rightarrow \infty .
$$

Notice that these estimates coincide with those valid for a solution of the homogeneous problem describing time-harmonic water waves in the presence of a fixed structure. It is known that conditions (20) and (18) are equivalent for the latter problem (see [6, §2.2.1] for the proof).

Definition 1. Let $\omega$ be an eigenvalue of problem (14)-(17), (20) for some structure $S$. If the corresponding eigensolution $(\phi, \zeta)$ has a nontrivial first component, then the velocity potential (13) is called a mode trapped by the structure $S$ (in fact, we have a family of trapped modes depending on the parameter $\alpha$ ), and $\omega$ is called a trapping frequency.

Remark 4. In Definition 1, it is essential that the component $\phi$ of an eigensolution be nontrivial. Indeed, if $\phi$ vanishes identically, then the problem reduces to a matrix eigenvalue problem whose eigenvectors must satisfy infinitely many orthogonality conditions depending on points of $B$. Presumably, the latter problem might have a solution only when rather tough restrictions are imposed on $B$. On the other hand, if an eigensolution $(\phi, 0)$ with nontrivial $\phi$ exists, then we have a mode trapped by a motionless structure which is, nevertheless, floating freely in waves.

Furthermore, relations (15)-(17) involve both $\omega$ and $\omega^{2}$, but using $\psi=\omega^{-1} \phi$ as the unknown function, we obtain the following spectral problem for $(\psi, \zeta)$ :

$$
\begin{aligned}
\nabla^{2} \psi & =0 \quad \text { in } \quad W, \quad \psi_{y}-\frac{\omega^{2}}{g} \psi=0 \quad \text { on } \quad F, \\
\frac{\partial \psi}{\partial n} & =\zeta \cdot N \quad \text { on } B, \quad \frac{\omega^{2}}{g} E \zeta-K \zeta=-\frac{\omega^{2}}{g} \int_{B} \psi N \mathrm{~d} s,
\end{aligned}
$$


which depends linearly on the parameter $\frac{\omega^{2}}{g}$. Another option of an unknown function is $\varphi=\frac{\omega}{g} \phi$, in which case both boundary conditions as well as the equations of motion again depend linearly on the same spectral parameter.

\section{§3. Auxiliary Results}

We describe a particular trapped-mode solution of the time-harmonic water-wave problem for a fixed structure (see [8] and [6, §4.2.2.3] for the details). This solution is used for constructing a solution of problem (14)-(17), (20). An essential moment is an application of the so-called inverse procedure, which was outlined at the end of $\S 1$. It replaces finding a solution for a prescribed structure by determining a water domain acceptable from a hydrodynamic point of view so that a given potential function delivers a solution of the spectral problem in this domain.

Consider the following functions:

$$
\begin{aligned}
& \phi_{0}(x, y)=\int_{0}^{\infty} \frac{k \mathrm{e}^{k \nu y}}{k-1}[\sin k(\nu x-\pi)-\sin k(\nu x+\pi)] \mathrm{d} k, \\
& \psi_{0}(x, y)=\int_{0}^{\infty} \frac{k \mathrm{e}^{k \nu y}}{k-1}[\cos k(\nu x-\pi)-\cos k(\nu x+\pi)] \mathrm{d} k,
\end{aligned}
$$

depending on a positive parameter $\nu$; the first of them will play the role of a given solution in our application of the inverse procedure. Notice that both integrals are understood as the usual improper integrals, because their integrands are bounded. Indeed, the singularities in the denominators coincide with the zeros of the numerators. It is clear that the functions $\phi_{0}$ and $\psi_{0}$ are harmonic in $\mathbb{R}_{-}^{2}$ and the second is conjugate to the first. Furthermore, $\phi_{0}$ and $\psi_{0}$ satisfy conditions (6) and (7), respectively.

In [6, pp. 177-178] it was shown that these functions attain finite values in

$$
\overline{\mathbb{R}_{-}^{2}} \backslash\{x= \pm d, y=0\} \text {, where } d=\pi / \nu \text {. }
$$

Moreover, the first of them can be written as follows:

$$
\phi_{0}(x, y)=(2 \nu)^{-1}\left[G_{x}(x, y ;-d, 0)-G_{x}(x, y ; d, 0)\right],
$$

where $G(x, y ; \xi, \eta)$ is the two-dimensional Green function of the time-harmonic waterwave problem (see [6, $\$ 1.2 .1]$, where the properties of $G$ were described). The latter representation guarantees that the boundary condition

$$
\partial_{y} \phi_{0}(x, 0)-\nu \phi_{0}(x, 0)=0
$$

is fulfilled for $x \neq \pm d$, and that the following estimates are valid:

$$
\phi_{0}(x, y)=O\left(\left[x^{2}+y^{2}\right]^{-1}\right), \quad\left|\nabla \phi_{0}(x, y)\right|=O\left(\left[x^{2}+y^{2}\right]^{-3 / 2}\right) \quad \text { as } \quad x^{2}+y^{2} \rightarrow \infty .
$$

We turn to the properties of the function $\psi_{0}$, which are of importance for constructing our example (see [6, pp. 178-179]). Since $\psi_{0}$ is an odd function of $x$, we formulate the required properties only for $x>0$. First, the trace $\psi_{0}(x, 0)$ has only one positive zero $x_{0} \in\left(\frac{2 \pi}{3 \nu}, \frac{\pi}{\nu}\right)$ and is negative on $\left(0, x_{0}\right)$. Second, $\psi_{0}(x, 0)$ increases monotonically from 0 to $+\infty$ on $\left[x_{0}, d\right)$, and decreases monotonically from $+\infty$ to 0 on $(d,+\infty)$.

Therefore, for every $b>0$, the level line

$$
B_{+}=\left\{(x, y) \in \overline{\mathbb{R}_{-}^{2}}: x>0, y<0, \psi_{0}(x, y)=b\right\}
$$

has the following properties (illustrated by the right part of Figure 1):

- the curve $B_{+}$connects two points $\left(x_{<}, 0\right)$ and $\left(x_{>}, 0\right)$ such that $x_{<} \in\left(x_{0}, d\right)$ and $x_{>} \in(d,+\infty)$

- the curve $B_{+}$violates the John condition (see Remark 2) on the left of the point $\left(x_{<}, 0\right)$. 


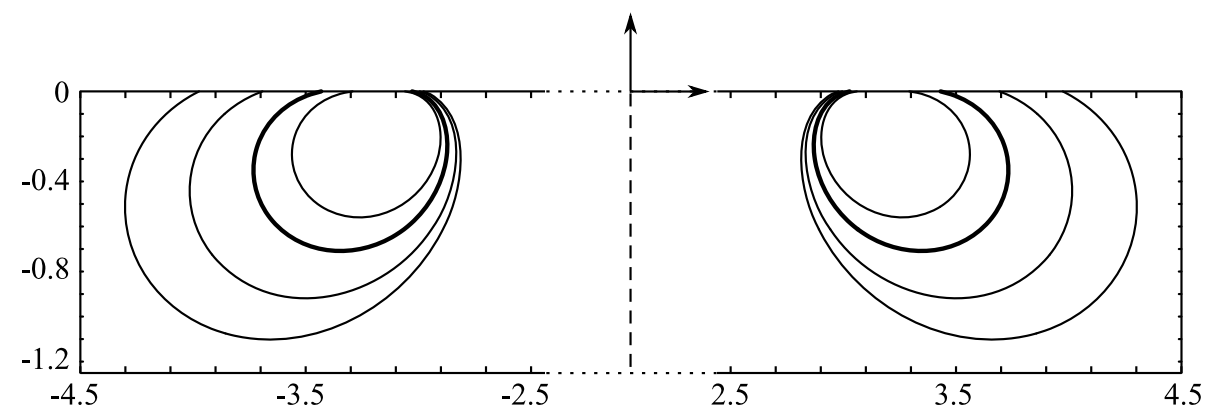

Figure 1. Level lines of $\psi_{0}$ plotted in nondimensional coordinates. In order to contract the figure's width, a reduced horizontal scale is applied on the interval $(-2.5,2.5)$.

The points $\left(x_{<}, 0\right)$ and $\left(x_{>}, 0\right)$ lie on the positive $x$-axis on either side of the point $(d, 0)$, where $\psi_{0}$ is infinite. Thus, $B_{+}$cuts out the latter point, and so $\phi_{0}$ has no singularity in the domain that lies in the quadrant $\{x>0, y<0\}$ outside $B_{+}$. Combining the latter property with the similar one for the level lines of $\psi_{0}$ in $\{x<0, y<0\}$, we arrive at the following statement.

Theorem 1. Let $\nu$ be a positive number, and let $B$ be the union of two level lines of the function $\psi_{0}$ with the following properties. One of these lines corresponds to a positive level and lies in the quadrant $\{x>0, y<0\}$, whereas the other lies in $\{x<0, y<0\}$ and corresponds to a negative level. (It is admissible that the absolute values of these two levels are not equal to each other, in which case $B$ is nonsymmetric.)

If a cylindrical structure has a cross section $S$ consisting of two domains placed between $B$ and the $x$-axis, then $\nu$ is an eigenvalue of the problem

$$
\nabla^{2} \phi=0 \quad \text { in } W, \quad \partial_{y} \phi-\nu \phi=0 \quad \text { on } F, \quad \frac{\partial \phi}{\partial n}=0 \quad \text { on } B,
$$

complemented by estimates (20) at infinity. The corresponding eigenfunction is $\phi_{0}$ defined with the help of the same value $\nu$.

Proof. It suffices to notice that $\phi_{0}$ satisfies the spectral boundary condition on $F$ because of (21). Furthermore, $\phi_{0}$ satisfies the homogeneous Neumann condition on $B$ because of the Cauchy-Riemann equations. Indeed, $B$ is the union of two level lines of the function $\psi_{0}$ conjugate to $\phi_{0}$.

Remark 5. There are infinitely many fixed structures for which $\nu$ is an eigenvalue of problem (23) and $\phi_{0}$ is the corresponding eigenfunction. One finds 16 examples of such structures in Fig. 1.

Two other kinds of examples of structures with the same property as in Theorem 1 can be obtained by adding one or two extra bodies. Their cross sections are also described in terms of the level lines of $\psi_{0}$. We have

$$
M_{-}=\min _{x \geq 0} \psi_{0}(x, 0)<0 \text { and } M_{+}=\max _{x \leq 0} \psi_{0}(x, 0)=-M_{-}>0,
$$

and we take arbitrary numbers $h_{-}$and $h_{+}$that belong to $\left(M_{-}, 0\right)$ and $\left(0, M_{+}\right)$, respectively. Then the level line $\psi_{0}(x, y)=h_{\mp}$ lies in $\{ \pm x>0, y<0\}$ and gives the wetted part of a body's cross section. Every such body together with a pair of bodies obtained in Theorem 1 forms a three-body trapping structure; combining the pair of Theorem 1 and the two bodies that correspond to $h_{-}$and $h_{+}$, we get a trapping structure of four bodies. 


\section{$\S 4$. MAIN THEOREM}

Let $b>0$, and let $B_{-}$denote the level line on which the function $\psi_{0}$ is equal to $-b$ (this line is symmetric about the $y$-axis to $B_{+}$introduced in $\S 3$ ). Therefore, $B_{0}=B_{+} \cup B_{-}$ bounds the cross section $S_{0}$ of the submerged part of some structure; we denote by $W_{0}$ and $F_{0}$ the cross sections of the corresponding water domain and free surface, respectively. Now we are in a position to formulate and prove our main result.

Theorem 2. Let $\omega_{0}$ and $b$ be arbitrary positive numbers. Let a freely floating structure satisfy conditions (i), (ii), and the three assumptions formulated prior to Proposition 1 (they are marked by bullets). Also, let the cross section of its submerged part be $S_{0}$ described above with the help of the function $\psi_{0}$ with $\nu$ equal to $\omega_{0}^{2} / g$.

Then the spectral problem (14) -(17), (20) has an eigensolution $\left(\phi_{0}, 0\right)$ in $W_{0}=\mathbb{R}_{-}^{2} \backslash \overline{S_{0}}$, and this solution corresponds to the eigenvalue $\omega_{0}$.

Proof. Theorem 1 guarantees that $\phi_{0}$ satisfies equation (14) in $W_{0}$, the boundary condition (15) is valid on $F_{0}=\partial \mathbb{R}_{-}^{2} \backslash \overline{S_{0}}$, and estimates (20) hold true for $\phi_{0}$ at infinity. Moreover, Theorem 1 shows that the boundary condition (16) is fulfilled on $B_{0}$ for the pair $\left(\phi_{0}, 0\right)$.

We turn to system (17) in which the integrals are calculated over $B_{0}$. The first and third equations of this system are valid for the pair $\left(\phi_{0}, 0\right)$, by Proposition 2 . Thus, it remains to verify the second equation (17), for which purpose we need to show that

$$
\int_{B_{0}} \phi_{0} n_{y} \mathrm{~d} s=0 .
$$

We write the second Green identity

$$
0=\int_{\partial\left(W_{0} \cap D_{R}\right)}\left(\phi_{0} \frac{\partial Y_{\nu}}{\partial n}-Y_{\nu} \frac{\partial \phi_{0}}{\partial n}\right) \mathrm{d} s
$$

for the harmonic functions $\phi_{0}$ and $Y_{\nu}=y+\nu^{-1}$; here $D_{R}$ is the disk of a sufficiently large radius $R$ centred at the origin, and $n$ is the inward normal on $\partial\left(W_{0} \cap D_{R}\right)$. Consider the integrals over the parts constituting this boundary. The boundary conditions (23) imply that the integral over $F_{0} \cap D_{R}$ vanishes and the integral over $B_{0}$ takes the form (24). In order to complete the proof, it suffices to observe that the integral over $\partial\left(\mathbb{R}_{-}^{2} \cap D_{R}\right)$ tends to zero as $R \rightarrow \infty$, which is a consequence of estimates (22).

Remark 6. It was pointed out in Remark 4 that the eigensolution $\left(\phi_{0}, 0\right)$ obtained in Theorem 2 describes a water-wave mode trapped by a motionless structure floating freely in waves and having $S_{0}$ as the cross section of its submerged part. Depending on the positive parameter $b$, such a structure is not unique and some examples that belong to the corresponding infinite family are shown in Figure 1. However, unlike the case of a fixed trapping structure, only those pairs of curves that are symmetric about the $y$-axis must be taken into account. Thus, only four examples of submerged parts of freely floating trapping structures are plotted in Figure 1 (one of them is singled out by bold lines), comparing the 16 fixed trapping structures.

In order to obtain another kind of motionless freely floating trapping structures, we can take into account what was said about fixed trapping structures in Remark 5. Namely, it is possible to complement any trapping structure described in Theorem 2 in such a way that the new structure be symmetric and its additional submerged parts be described by the level lines $\psi_{0}(x, y)=h$ and $\psi_{0}(x, y)=-h$, where $h \in\left(0, M_{+}\right)$. Since $h$ is arbitrary within the interval indicated, we see that the freely floating trapping structures that have four submerged parts form a family depending on two real parameters. 
Remark 7. By the uniqueness theorem of John (see Remark 3), sufficiently large values $\omega$ cannot be eigenvalues of the spectral problem (14)-(18). On the contrary, Theorem 2 says that any $\omega$ can serve as an eigenvalue for properly constructed structures. However, these structures violate both conditions essential for John's theorem; namely, they consist of more than one body and the geometric condition of John is not valid for them.

\section{REFERENCES}

[1] F. John, On the motion of floating bodies. I, Comm. Pure Appl. Math. 2 (1949), 13-57. MR0032328 $(11: 279 \mathrm{~b})$

[2] J. T. Beale, Eigenfunction expansions for objects floating in an open sea, Comm. Pure Appl. Math. 30 (1977), 283-313. MR0670432 (58:32320)

[3] F. John, On the motion of floating bodies. II, Comm. Pure Appl. Math. 3 (1950), 45-101. MR0037118 (12:214h)

[4] P. McIver and M. McIver, Trapped modes in the water-wave problem for a freely floating structure, J. Fluid Mech. 558 (2006), 53-67. MR2261775 (2007i:76012)

[5] C. J. Fitzgerald and P. McIver, Passive trapped modes in the water wave problem for a floating structure, J. Fluid Mech. 657 (2010), 456-477. MR2671605

[6] N. Kuznetsov, V. Maz'ya, and B. Vainberg, Linear water waves. A mathematical approach, Cambridge Univ. Press, Cambridge, 2002. MR 1925354 (2003i:76009)

[7] M. McIver, An example of non-uniqueness in the two-dimensional linear water-wave problem, J. Fluid Mech. 315 (1996), 257-266. MR1403701 (97h:76016)

[8] O. Motygin and N. Kuznetsov, Non-uniqueness in the water-wave problem: an example violating the inside John condition, Proceedings of the 13th International Workshop on Water Waves and Floating Bodies, Delft Univ. Technology, 1998, pp. 107-110. Available at http://www.iwwwfb.org/ Abstracts/iwwwfb13/iwwwfb1328.pdf

[9] L. Euler, Principia motus fluidorum, Novi Commentarii Academiae Scientiarum Imperialis Petropolitanae. T. VI, 1761, pp. 271-311. English translation available at http:// www.math.dartmouth.edu/ euler/docs/translations/E258.pdf

[10] D. W. Fox and J. R. Kuttler, Sloshing frequencies, Z. Angew. Math. Phys. 34 (1983), 668-696. MR0723140 (84k:76027)

[11] S. A. Nazarov and J. Taskinen, On the spectrum of the Steklov problem in a domain with a peak, Vestnik S.-Peterburg. Univ. Ser. 1 2008, vyp. 1, 56-65; English transl., Vestnik St. Petersburg Univ. Math. 41 (2008), 45-52. MR2406898 (2010b:35065)

[12] P. É. Appell, Traité de mécanique rationelle. T. 3, Gauthier-Villars, Paris, 1932.

Institute of Mechanical Engineering Problems, Russian Academy of Sciences, Bol'shoŭ Pr. 61, St. Petersburg 199178, Russia

E-mail address: nikolay.g.kuznetsov@gmail.com

Received 5/APR/2010

Originally published in English 